\title{
A Software for the Evaluation of Winding Factor Harmonic Distribution in High Efficiency Electrical Motors and Generators
}

\author{
A. O. Di Tommaso, F. Genduso, R. Miceli, Member, IEEE
}

\begin{abstract}
In this paper a software developed for the determination of winding connections, the calculation of winding factors and the evaluation of their harmonic distribution of three and two phase windings is presented by using as input data simply the number of slots, pole pairs and phases. The software is implemented as a function in MATLAB environment. By means of some examples, covering the most relevant winding types, the capabilities of this software will be shown. Particularly, integer and fractional single and double layer winding can be treated. Some examples are presented and briefly discussed. The connections, the winding factor harmonic distribution, the electro-motive force (e.m.f) THD calculations results are shown together with an example on how is possible to determine the e.m.f. induced in a winding from the air gap flux distribution and to estimate the differential leakage inductance.
\end{abstract}

Index Terms-winding, winding factor, winding connections, harmonics.

\section{LIST OF SYMBOLS}

$N$

$p$

$r$

$t$

$\nu$

$m$

$q$

$k_{d \nu}$

$k_{w \nu}$

$k_{s l \nu}$

$k_{s k \nu}$

$k_{c \nu}$

$E_{s}$

$C_{i, j}$

$S_{i, j}$ number of slots;

number of pole pairs;

star pitch;

greatest common divider (GCD) between $N$ and $p$ $t=G C D(N, t)$;

harmonic order;

number of phases;

number of slots per pole and per phase;

distribution factor at the $\nu$-th harmonic order;

group factor at the $\nu$-th harmonic order;

slot opening factor at the $\nu$-th harmonic order;

skewing factor at the $\nu$-th harmonic order;

winding connection factor at the $\nu$-th harmonic order;

e.m.f. induced in a group of slot conductors;

phase component of $E_{s}$;

quadrature component of $E_{s}$;

Antonino Oscar Di Tommaso is with the Department of Energy, Information engineering and Mathematical models (DEIM) - University of Palermo, Palermo, Italy, e-mail: antoninooscar.ditommaso@unipa.it.

Fabio Genduso is with the Department of Energy, Information engineering and Mathematical models (DEIM) - University of Palermo, Palermo, Italy, e-mail: fabio.genduso@unipa.it.

Rosario Miceli is with the Department of Energy, Information engineering and Mathematical models (DEIM) - University of Palermo, Palermo, Italy, e-mail: rosario.miceli@unipa.it.

This work was financially supported by MIUR - Ministero dell'Istruzione dell'Università e della Ricerca (Italian Ministry of Education, University and Research) and by SDESLab (Sustainable Development and Energy Saving Laboratory) of the University of Palermo.
$N_{c} \quad$ number of coil sides per phase;

$\nu_{x} \quad$ maximum harmonic order;

$b_{s} \quad$ is the slot opening expressed as a fraction of the slot pitch;

$Z_{s k} \quad$ number of skewing sections;

$\sigma_{0} \quad$ differential leakage factor.

\section{INTRODUCTION}

T ONG-TERM operations of electrical motors and generators are expected to have high energetic efficiency as one of the most important goals to reach [1], [2], [3], [4]. In this paper a software strictly developed for the purpose of winding factor harmonic distribution evaluation is presented. As known winding factors, and consequently the harmonic content of the electrical quantities involving such machines, are determined by the type of winding employed in their construction. Particularly, the harmonic distortion of voltages, currents, flux densities, etc., in electrical machines -which depend on the type of winding- affect in a significant manner both the dynamic and steady state performances and their efficiency, by introducing, for example, asynchronous, synchronous and torsional torques, axial and radial forces producing vibrations (and noise), and, last but not least, producing an increase in copper and iron losses. It is, therefore, important to have a clear overview on the winding factor harmonic distribution characterizing an electrical machines indeed just in the design phase, i.e. when the right type of winding is to be chosen [5], [6]. In this sense the proposed software is conceived as an aid to simplify and accelerate the design procedures in electrical machine windings. The software is also capable to represent numerically the winding connections, in the case of single and double layer structures, and to perform some useful calculation about phase leakage inductances. Some examples will be presented in this paper to show and to explain the capabilities of the software, dealing both with integer and fractional winding types.

In section II the mathematical expressions of the winding factors are presented and the software procedure of the winding connection construction is briefly outlined. In section III some examples are presented and discussed.

\section{Winding FACTORS AND PROCEDURE FOR WINDING CONNECTIONS}

In this section the mathematical aids used to determine the winding connections, the method of winding factors calcula- 
tion and the determination of their harmonic distribution are presented.

As known from the theory of electrical machines [7], [8], [9], [10] each conductor or group of conductors in a slot is characterized by a particular phasor of the induced e.m.f.. All these phasors can be represented in a polar diagram called slot star or e.m.f. star. If we define $t$ as the greatest common divider between the slot number $N$ and the pole pair number $p, t$ slot e.m.f. phasors will have the same phase and a star of

$$
S=\frac{N}{t}
$$

phasors with different phases will be obtained. The phase angle between e.m.f. of two adjacent phasors within the slot star relative to the fundamental wave is, therefore,

$$
\alpha^{\prime}=\frac{t}{N} 360^{\circ}
$$

while the phase angle between e.m.f. of adjacent slots is

$$
\alpha=\frac{p}{N} 360^{\circ}=\frac{p}{t} \alpha^{\prime} .
$$

For all other harmonic orders it is sufficient to multiply both $\alpha$ or $\alpha^{\prime}$ by the harmonic order coefficient $\nu$.

With the help of (3) it is possible to determine a correspondence between the slot and star phasors numbering. Therefore, if the slots are numbered in the order $Z=1,2,3, \ldots$ the phasors of the star will be numbered by jumping over $p / t-1$ star phasors, meaning that adjacent slot e.m.f. have a distance of $p / t$ steps in the star.

An important quantity for the determination of a e.m.f. star is the star pitch defined as

$$
r=\frac{g \frac{N}{t}+1}{\frac{p}{t}}=\text { integer }
$$

with $g=0,1,2, \ldots$

By applying (4) it is possible to evaluate the slot e.m.f. star and from this, with the help of a procedure similar to that used to realize the Tingley's scheme [8], the winding connection order. The last is expressed as a matrix of numbers with each row corresponding to the phase $a, b$ and $c$. Each element of a row is referred to a slot number and the number sequence of each row represents how the coil sides (located within each slot) are to be connected in series to form the phase winding section

$$
\operatorname{Conn}_{h, i}=\left[\begin{array}{ccccc}
a_{1} & a_{2} & a_{3} & \ldots & a_{N / m} \\
b_{1} & b_{2} & b_{3} & \ldots & b_{N / m} \\
c_{1} & c_{2} & c_{3} & \ldots & c_{N / m}
\end{array}\right]
$$

Tables I, II and III summarize the procedure of building the connection matrix $\operatorname{Conn}_{h, i}$ in the case of a single layer fractional winding.

Table I

CONNECTION SCHEME OF A FRACTIONAL SLOT WINDING WITH N=24, $\mathrm{P}=5, \mathrm{R}=5$

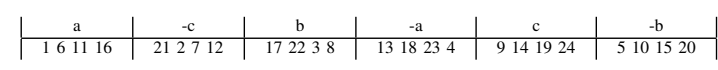

Table II

PER-PHASE CONNECTION SCHEME OF A FRACTIONAL SLOT WINDING WITH $\mathrm{N}=24, \mathrm{P}=5, \mathrm{R}=5$

\begin{tabular}{c|c|c|c|c|} 
& \multicolumn{2}{|c|}{$\mathrm{a}$} & $\mathrm{b}$ & $\mathrm{c}$ \\
\hline+ & 161116 & 172238 & 9141924 \\
- & 1318234 & 5101520 & 212712
\end{tabular}

Table III

CONNECTION MATRIX FOR $\mathrm{N}=24, \mathrm{P}=5, \mathrm{R}=5$

\begin{tabular}{c||c|c|c|c|c|c|c|c}
$\mathrm{a}$ & 1 & 6 & 11 & 16 & -13 & -18 & -23 & -4 \\
\hline $\mathrm{b}$ & 17 & 22 & 3 & 8 & -5 & -10 & -15 & -20 \\
\hline $\mathrm{c}$ & 9 & 14 & 19 & 24 & -21 & -2 & -7 & -12
\end{tabular}

Once the winding connections are defined it is possible to determine all the winding factors as it will be explained below.

Traditionally, in literature [7], [8], [9], [10] many formulas have been proposed for the calculation of these factor.

In the case of a group of $S$ coils, all with a diametrical coil pitch, the distribution factor (group factor) of the $\nu$-th harmonic is [11]

$$
k_{d \nu}=\frac{\sin \left(\nu S \frac{\pi p}{N}\right)}{S \sin \left(\nu \frac{\pi p}{N}\right)}=\frac{\sin \left(\nu \frac{\pi}{2 m}\right)}{q \sin \left(\nu \frac{\pi}{2 m q}\right)}
$$

where $m$ is the number of phases of the winding and $q$ the number of slots per pole and per phase

$$
q=\frac{N}{2 p m} .
$$

If the coil pitch is shortened then the resulting winding factor $k_{w \nu}$ is obtained by multiplying $k_{d \nu}$ by the shortening pitch factor defined as

$$
\begin{gathered}
k_{p v}=\sin \left(\nu \frac{\pi}{2}\right) \cdot \sin \left(\nu \frac{2 p \eta}{N} \frac{\pi}{2}\right) \quad \text { for } \nu \text { odd } \\
k_{p v}=-\cos \left(\nu \frac{\pi}{2}\right) \cdot \sin \left(\nu \frac{2 p \eta}{N} \frac{\pi}{2}\right) \quad \text { for } \nu \text { even } \\
k_{w \nu}=k_{p \nu} \cdot k_{d \nu} .
\end{gathered}
$$

where $\eta$ is the coil pitch expressed in terms of slot numbers. Normally the shortening pitch factor can be calculated applying (8) when $q$ is an integer, but, when dealing with fractional slot windings, in which the spatial distribution of the magnetic flux density has no half period symmetry, also the even order harmonics must be considered [7], [9], [10]. This aspect can often yield to tedious calculations.

In case of complex winding configurations, as for example with fractional slot windings where the e.m.f.s do not show symmetries (see Fig. 1 a) and b)), the winding factors will be made up by more complex formulas [9], [10], [7]. In order to gain generality and to simplify the algorithm, the winding factor definition is considered as the ratio between the geometrical and the algebraic sum of the e.m.f. induced in a winding section: 


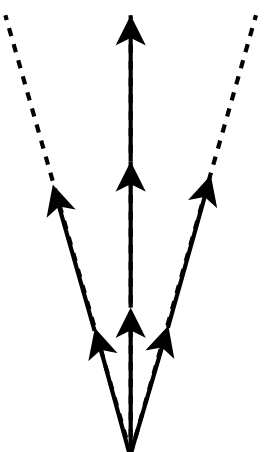

a)

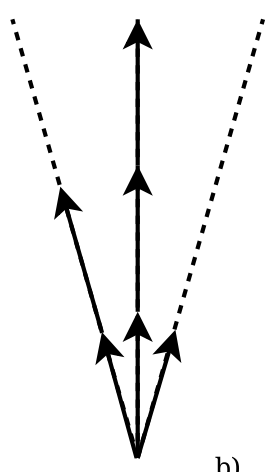

b)
Figure 1. Difference between a) symmetrical and b) unsymmetrical phase e.m.f. systems.

$$
k_{w \nu}=\frac{\sum_{\nu=1}^{\nu_{x}} \sum_{j=1}^{N} \sqrt{C_{i, j}^{2}+S_{i, j}^{2}}}{N_{c} \cdot E_{s}}
$$

where

$$
\begin{aligned}
& C_{i, j}=k \cdot E s \cdot \cos \left(i \cdot 2 \pi \cdot \frac{\text { Conn }_{h, j}}{N}\right) \\
& S_{i, j}=k \cdot E s \cdot \sin \left(i \cdot 2 \pi \cdot \frac{\operatorname{Conn}_{h, j}}{N}\right)
\end{aligned}
$$

and

$$
k=\left\{\begin{array}{ll}
+1 & \text { if } \operatorname{Conn}_{h, j}>0 \\
-1 & \text { if } \operatorname{Conn}_{h, j}<0
\end{array} .\right.
$$

In (12), (13) and (14) $h$ can be set equal $a$ or $b$ or $c$ indifferently. It is to be noted that (11) is valid for the determination of the resultant winding factor also in the case of coil pitch shortening; as a matter of fact, information about pitch shortening is already contained in the matrix $\operatorname{Conn}_{h, j}$. Only if one will take also the effects of slot openings and of pole or slot skewing into account, further correction factors must be introduced, i.e., respectively:

$$
k_{s l \nu}=2 \cdot \frac{\sin \left(\nu \cdot b_{s} \cdot \frac{\pi}{N}\right)}{\nu \cdot b_{s} \cdot \frac{2 \pi}{N}}
$$

and

$$
k_{s k \nu}=\frac{\sin \left(\nu \cdot \frac{\pi}{N}\right)}{Z_{s k} \cdot \sin \left(\nu \cdot \frac{\pi}{Z_{s k} N}\right)}
$$

where $Z_{s k}$ is the number of skewing sections in the case of a discrete skewing (see Fig. 2). Furthermore, taking the winding phase connections into account (concatenated e.m.f.) the following factor is introduced:

$$
k_{c \nu}=\sin \left(\nu \cdot \frac{\pi \cdot p}{m}\right) .
$$

In this case (11) becomes

$$
k_{w \nu}=\frac{\sum_{\nu=1}^{\nu_{x}}\left(k_{c \nu} \cdot k_{s l \nu} \cdot k_{s k \nu} \cdot \sum_{j=1}^{N} \sqrt{C_{i, j}^{2}+S_{i, j}^{2}}\right)}{N_{c} \cdot E_{s}}
$$

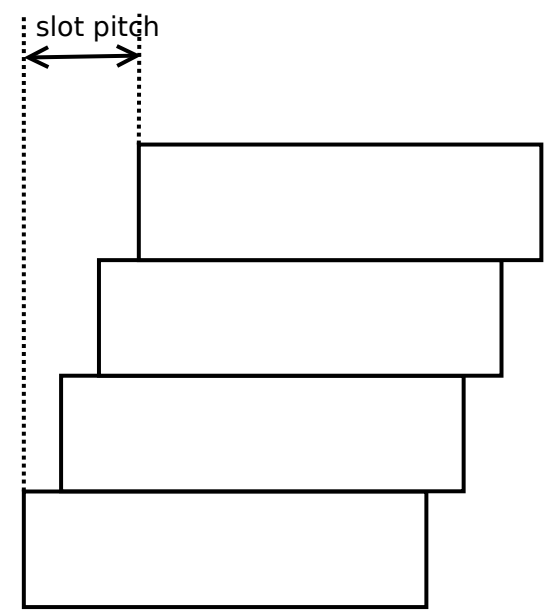

Figure 2. To clear the meaning of skewing sections. Here the skewing is made by 4 sections $\left(Z_{s k}=4\right)$.

with $\nu=1,2,3, \ldots, \nu_{x}$, which is the general formula for winding factor calculation implemented in the MATLAB program here presented.

Equation (18) can also be used to compute the so called differential leakage factor as [9], [12], [8], [7]

$$
\sigma_{0}=\sum_{\nu=1}^{p-1}\left(\frac{k_{w \nu}}{\nu k_{w p}}\right)^{2}+\sum_{\nu=p+1}^{\nu_{x}}\left(\frac{k_{w \nu}}{\nu k_{w p}}\right)^{2} .
$$

The developed MATLAB software mainly consist of three subroutines: the first one accepts as inputs the $N, p$ and $m$ values and gives as output the connection matrix $\operatorname{Conn}_{\nu, j}$; the second one accepts as inputs a single rows of the connection matrix and $p$, and gives as an output the $\sigma_{0}$ differential leakage factor; the third one accept as inputs a row of the connection matrix, the number of pole pairs and the radial component of the flux density along the air gap, giving as outputs the induced e.m.f. in the winding and the THD factor of the same e.m.f.. The next section illustrates some examples, referred to 4 particular cases of stator windings, whose results were obtained with the developed software. The first three examples involve the first and the second subroutines, therefore the results are independent from the machine type, while the fourth example is related to the case of an particular IPM machine.

\section{EXAMPLES}

In this section some examples used to test the program are presented and discussed. Particularly, first the connection matrix will be shown and then the winding factor spectral distribution will be analyzed.

Example 1. Three phase double layer winding with $N=27$ slots, $p=3, q=1+1 / 2$.

By introducing these data as input for the MATLAB function the following $3 x 6$ connection matrix is obtained.

$$
\left[\begin{array}{llllll}
1 & -5 & 2 & -6 & -6 & 1 \\
4 & -8 & 5 & -9 & -9 & 4 \\
7 & -2 & 8 & -3 & -3 & 7
\end{array}\right]
$$


The matrix has a reduced dimension because this is a base winding type, i.e. the complete winding is obtained by repeating these connections $p=3$ times.

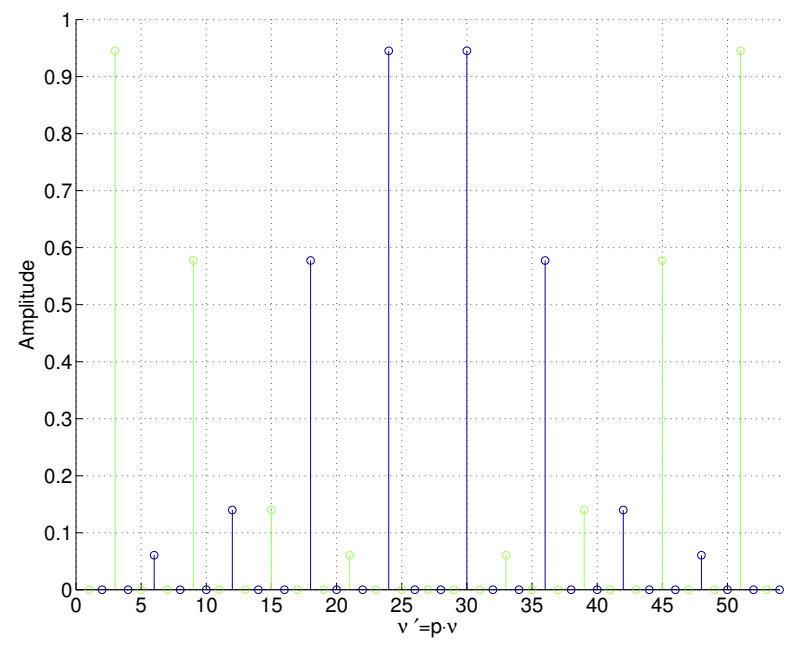

Figure 3. Winding factor spectrum for $N=27$ and $p=3$.

In Fig. 3, 4 and 5 the spectral distributions of the winding factors are represented in the order: $k_{w \nu}, k_{c \nu} \cdot k_{w \nu} \cdot k_{s l \nu}$ and $k_{c \nu} \cdot k_{s l \nu} \cdot k_{s k \nu} \cdot k_{w \nu}$, with $b_{s}$ equal to half the slot width and $Z=4$ skewing sections.

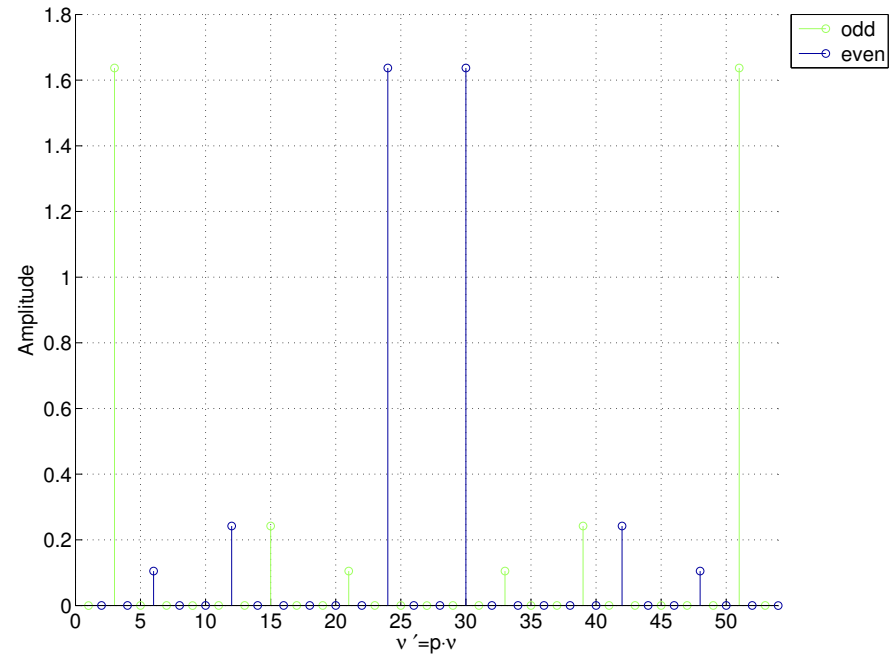

Figure 4. Resultant winding factor spectrum for $N=27$ and $p=3\left(k_{r e s}=\right.$ $\left.k_{c \nu} \cdot k_{s l \nu} \cdot k_{w \nu}\right)$.

The winding connection scheme is represented in Fig. 6.

Example 2. Three phase double layer winding with $N=27$ slots, $p=2, q=2+1 / 4$.

By introducing these data as input for the MATLAB function the connection matrix is obtained. For simplicity only the first row of this matrix is shown, which is used to determine the winding factor spectral distribution by means of (11) or (18).

$$
\left[\begin{array}{cccccccccc}
1 & -7 & 15 & -21 & 2 & -8 & 16 & -22 & 3 & \cdots \\
-9 & -8 & 12 & -22 & 1 & -9 & 14 & -23 & 2 &
\end{array}\right]
$$

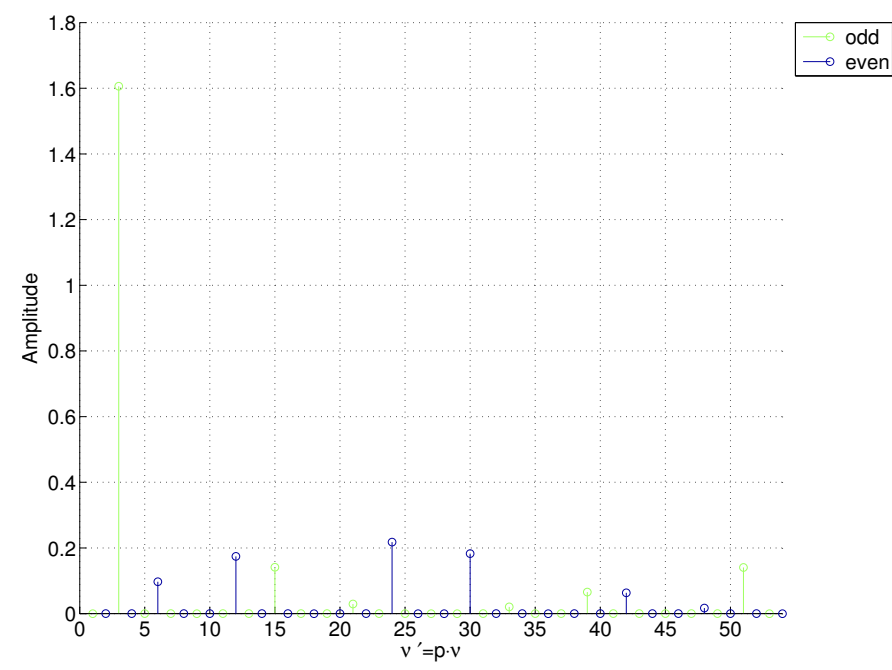

Figure 5. Resultant winding factor spectrum for $N=27$ and $p=3$ ( $k_{r e s}=$ $\left.k_{c \nu} \cdot k_{s k \nu} \cdot k_{s l \nu} \cdot k_{w \nu}\right)$.

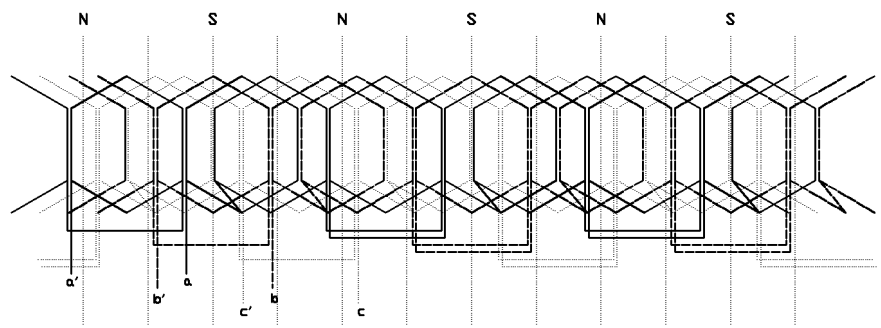

Figure 6. Three phase double layer winding of the IPMSM for $N=27$ and $p=3$.

In Fig. 7, 8 and 9 the spectral distributions of the winding factors are represented in the order: $k_{w \nu}, k_{w \nu} \cdot k_{s l \nu}$ and $k_{s l \nu}$. $k_{s k \nu} \cdot k_{w \nu}$, with $b_{s}$ equal to half the slot width and $Z=4$ skewing sections.

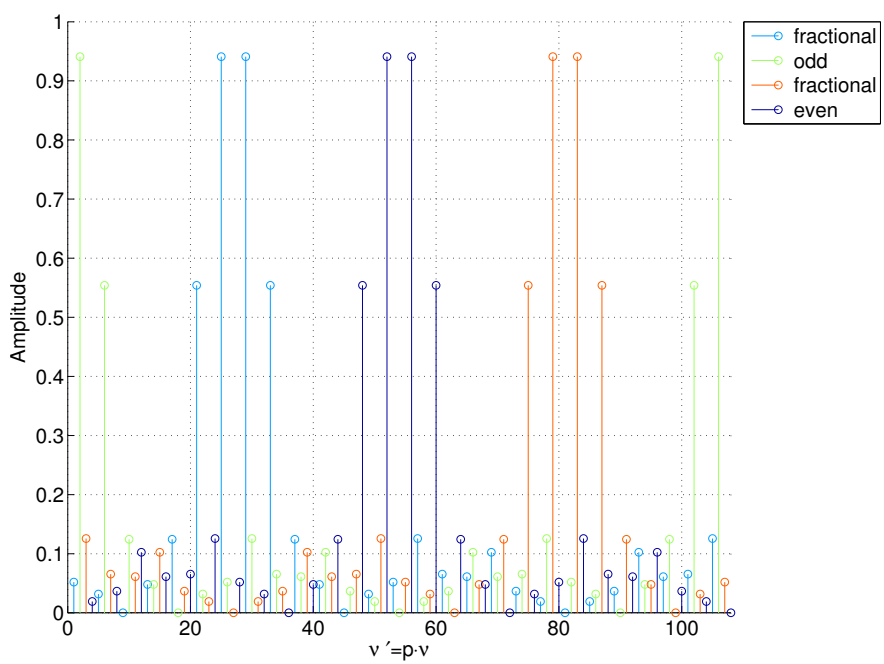

Figure 7. Winding factor spectrum for $N=27$ and $p=2$.

Example 3. Three phase double layer winding with $N=27$ slots, $p=4, q=1+1 / 8$.

By introducing these data as input for the MATLAB func- 


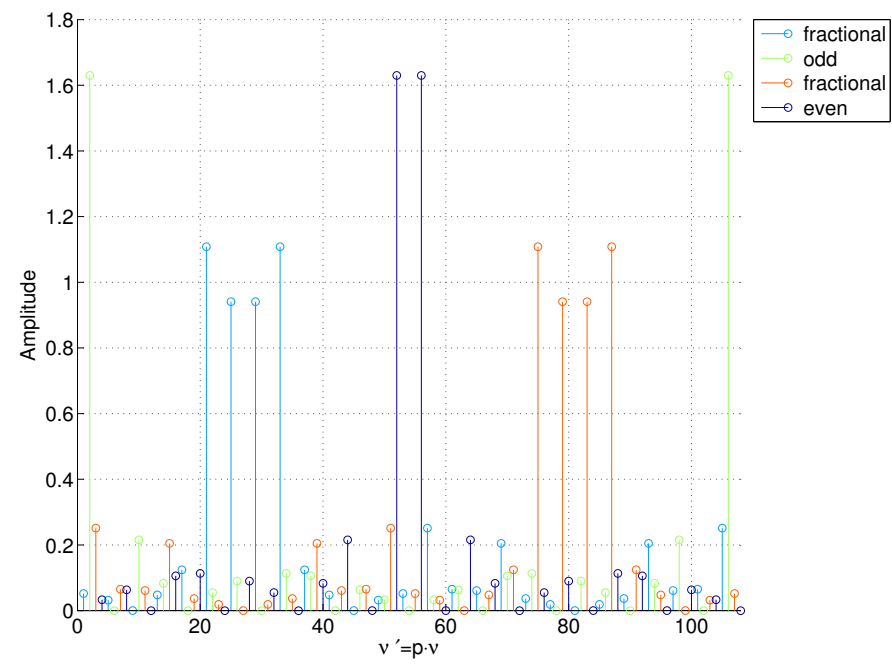

Figure 8. Resultant winding factor spectrum for $N=27$ and $p=2\left(k_{r e s}=\right.$ $\left.k_{c \nu} \cdot k_{s l \nu} \cdot k_{w \nu}\right)$.

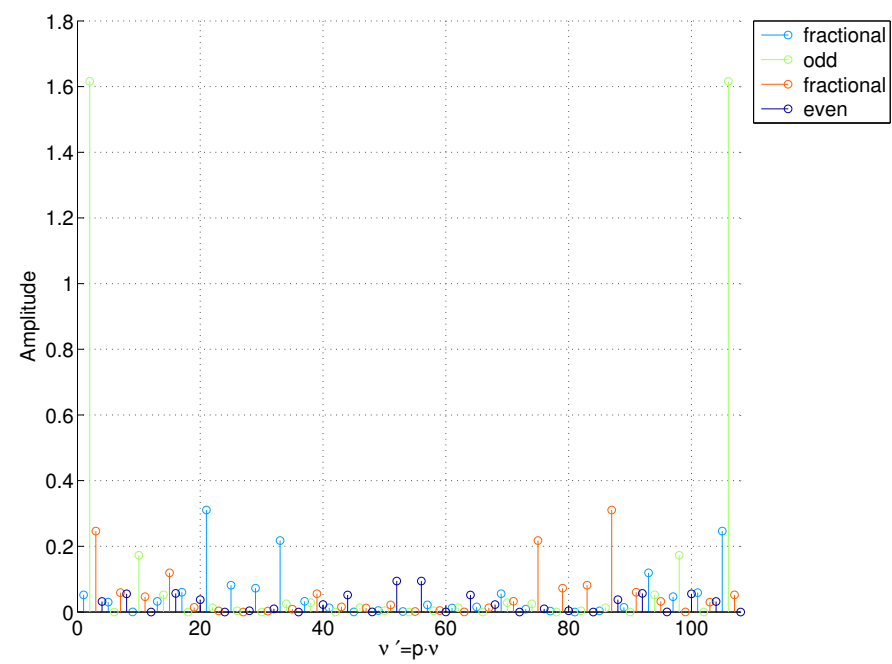

Figure 9. Resultant winding factor spectrum for $N=27$ and $p=2\left(k_{r e s}=\right.$ $\left.k_{c \nu} \cdot k_{s k \nu} \cdot k_{s l \nu} \cdot k_{w \nu}\right)$.

tion the connection matrix is obtained. For simplicity only the first row of this matrix is shown, which is used to determine the winding factor spectral distribution by means of (11) or (18).

$$
\left[\begin{array}{cccccccccc}
1 & -4 & 8 & -11 & 15 & -18 & 22 & -25 & 2 & \cdots \\
-5 & -18 & 21 & -25 & 1 & -5 & 8 & -12 & 15 &
\end{array}\right]
$$

For brevity in this example only the spectral distributions of the resultant winding factor is represented in Fig. 10 (i.e. $k_{s l \nu} \cdot k_{s k \nu} \cdot k_{w \nu}$, with $b_{s}$ equal to half the slot width and $Z=4$ skewing sections).

Example 4. Regarding Example 1 here an other example is reported in order to show how the air-gap e.m.f. of an electrical machine can be estimated by knowing machine parameters and its spatial distribution of the air-gap flux density. The machine is a IPMSM whose main parameters are listed in Table IV and Table $\mathrm{V}$ while a cross section of the machine is depicted in Fig. 11.

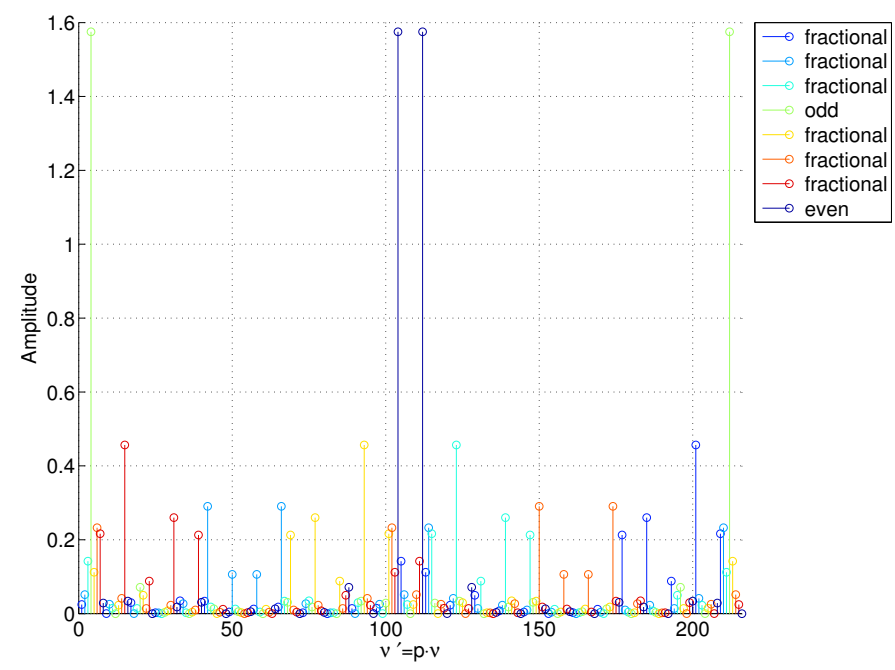

Figure 10. Resultant winding factor spectrum for $N=27$ and $p=4\left(k_{r e s}=\right.$ $\left.k_{c \nu} \cdot k_{s k \nu} \cdot k_{s l \nu} \cdot k_{w \nu}\right)$.

Table IV

RATED VALUES OF THE IPMSM.

\begin{tabular}{lr}
\hline Item [unit] & Specification \\
\hline \hline Rated speed [rpm] & 4000 \\
Rated current [A] & 3.6 \\
Rated phase voltage [V] & 77 \\
Rated torque [Nm] & 1.8 \\
Number of poles & 6 \\
Number of conductors per slot & 20
\end{tabular}

Table V

STRUCTURE PARAMETERS OF IPMSM. ALL MEASUREMENTS ARE EXPRESSED IN MM.

\begin{tabular}{lr}
\hline Item & Specification \\
\hline \hline External stator diameter & 81 \\
Internal stator diameter & 49.6 \\
External rotor diameter & 48 \\
Internal rotor diameter & 18.46 \\
Axial rotor length & 59 \\
PM width & 13.45 \\
PM thickness & 3 \\
Air-gap thickness & 0.8 \\
Slot depth & 9.2 \\
Slot mean width & 4 \\
Stator screw hole diameter & 2 \\
Rotor screw hole diameter & 5 \\
Shaft flux barrier thickness & 1 \\
\hline
\end{tabular}

Fig. 12 represents the spatial distribution of the radial component of flux density along the air-gap, which is produced by the interaction between the permanent magnet field and the armature reaction field produced at full load. Despite the heavy distortion produced by the slot harmonics, by the armature reaction and the saturation of iron, the induced e.m.f. shape maintains quite sinusoidal, due to the effect of winding factors, as shown in Fig. 13. The quality of e.m.f. is proved by THD\% $=0.85$, while, applying (19), the differential leakage factor is $\sigma_{0}=0.0036$.

\section{CONCLUSIONS}

In this paper a software written in MATLAB language was synthetically presented and tested by means of 4 examples. 


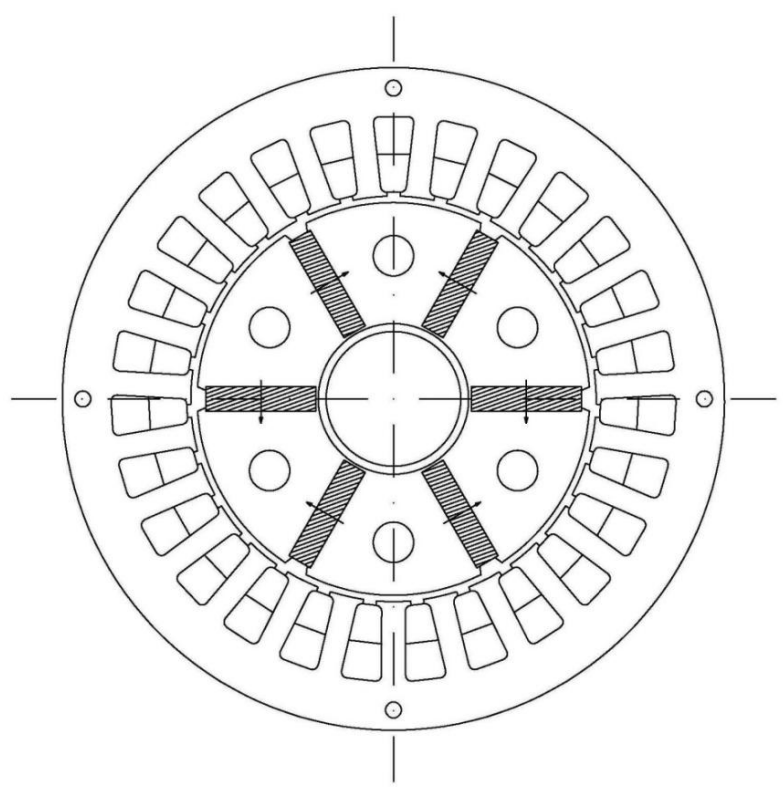

Figure 11. Cross section of the IPMSM.

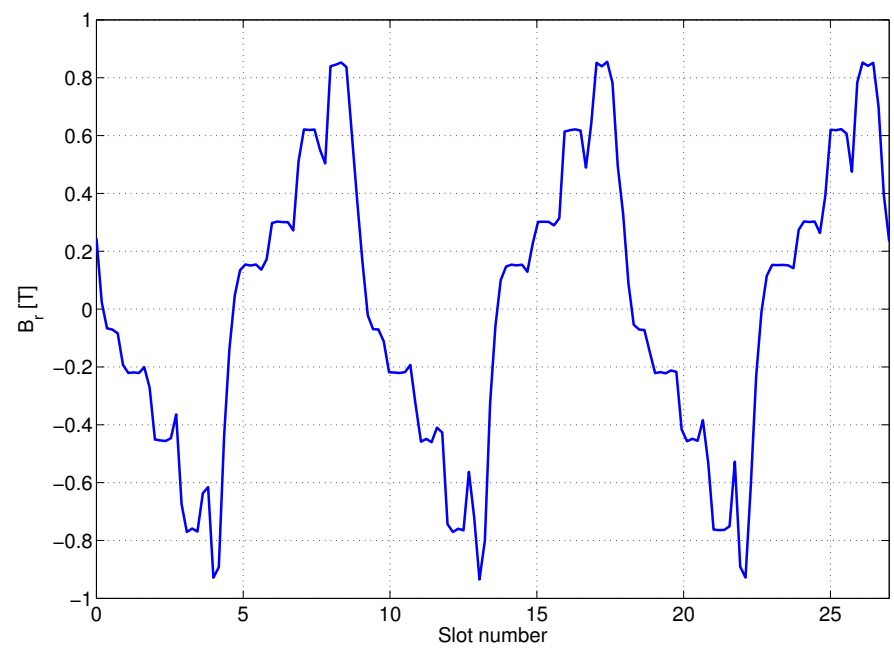

Figure 12. IPMSM radial component of the air-gap flux density.

The software can be used as a winding design tool as well as a winding analysis tool, being it capable to:

1) determine schematically the connection order of the coil sides located in machine slots;

2) determine the spectral distribution of winding factors;

3) to estimate the shape of in a winding induced e.m.f., from air-gap flux density;

4) to estimate the THD and the differential leakage factor of both integer and fractional slot windings.

For the determination of the winding connections only few elements are required as: the knowledge of the numbers of slots, of pole pairs, the number of phases and the type of winding (single or double layer). On the other hand for the e.m.f. induced in a winding the knowledge of more data is necessary, such as the angular speed, the number of conductors in a slot, the spatial distribution of flux density, etc..

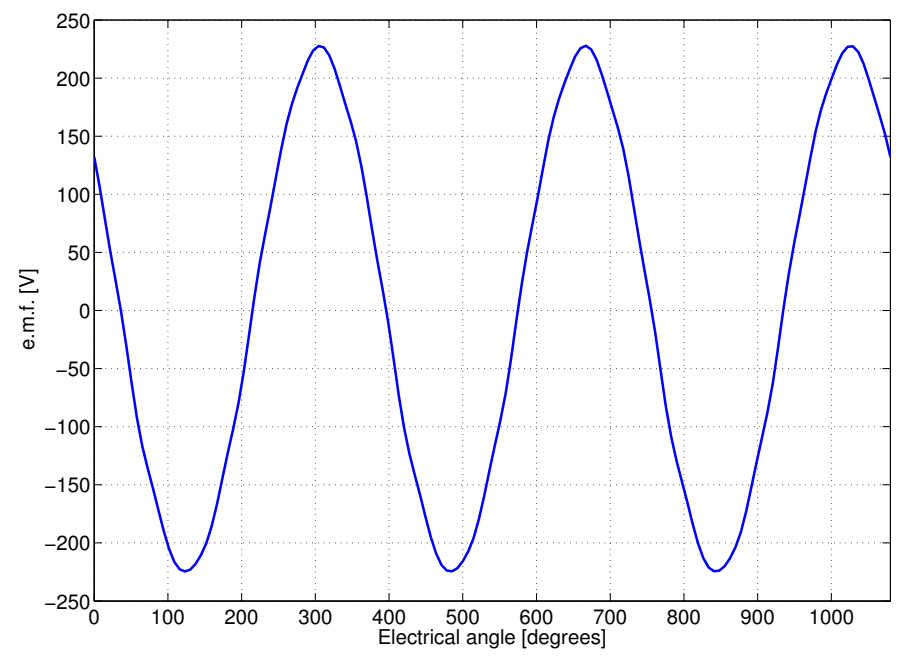

Figure 13. IPMSM induced e.m.f. at an angular speed of $4000 \mathrm{rpm}$.

\section{ACKNOWLEDGMENT}

The Authors wish to thank all the collaborators and the staff of SDESLab (Sustainable Development and Energy Savings Laboratory)-University of Palermo- and Emergenzamatic S.r.1..

\section{REFERENCES}

[1] C. Cavallaro, A. O. Di Tommaso, R. Miceli, A. Raciti, G. Ricco Galluzzo, and M. Trapanese, Efficiency Improvement of Inverter-Fed Permanent Magnet Synchronous Motors, pp. 490-497. Energy Efficiency in Motor Driven Systems, Springer Verlag, 1 ed., 2003.

[2] C. Cavallaro, A. O. Di Tommaso, R. Miceli, A. Raciti, G. Ricco Galluzzo, and M. Trapanese, "Analysis a DSP Implementation and Experimental Validation of a Loss Minimization Algorithm Applied to Permanent Magnet Synchronous Motor Drives," in The 29th Annual Conference of the IEEE Industrial Electronics Society, 2003. IECON '03., pp. 312-317, IEEE, IEEE, 2003.

[3] C. Cavallaro, A. O. Di Tommaso, R. Miceli, A. Raciti, G. Ricco Galluzzo, and M. Trapanese, "Efficiency Enhancement of Permanent Magnet Synchronous Motor Drives by on-Line Loss Minimization Approaches," IEEE Transactions on Industrial Electronics, vol. Volume 52, pp. 1153-1160, Aug. 2005.

[4] A. O. D. Tommaso, R. Miceli, G. R. Galluzzo, and M. Trapanese, "Efficiency Control for Permanent Magnet Synchronous Generators," in IEEE International Conference on Industrial Technology, ICIT 2006., pp. 2079-2084, IEEE, IEEE, December 2006.

[5] A. O. Di Tommaso, G. Ricco Galluzzo, and R. Miceli, "Improvement of IPMSM Performance through a Mixed Radial-tangential Rotor Structure," in IEEE International Symposium on Industrial Electronics (ISIE), (Bari, Italy), pp. 1327-1332, IEEE, 2010.

[6] Y. Gritli, A. O. Di Tommaso, F. Filippetti, R. Miceli, C. Rossi, and A. Chatti, "Investigation of motor current signature and vibration analysis for diagnosing rotor broken bars in double cage induction motors," in International Symposium on Power Electronics, Electrical Drives, Automation and Motion (SPEEDAM), (Sorrento, Italy), pp. 1360-1365, 20-22 June 2012.

[7] R. Richter, Lehrbuch der Wicklungen elektrischer Maschinen. Wissenschaftliche Bücherei, Verlag und Druck G. Braun, 1952.

[8] H. Sequenz, Die Wicklungen elektrischer Maschinen, vol. 1. Springer Verlag, 1950.

[9] G. Müller, K. Vogt, and B. Ponick, Berechnung elektrischer Maschinen. Elektrische Maschinen, Wiley-VCH Verlag GmbH \& Co KGaA, 6 ed., 2008.

[10] G. Müller and B. Ponick, Theorie elektrischer Maschinen. Elektrische Maschinen, Wiley-VCH Verlag GmbH \& Co KGaA, 6 ed., 2009.

[11] R. Richter, Allgemeine Berechunungselemente - Gleichstrommaschinen, vol. 1 of Elektrische Maschinen. Basel, Stuttgart: Birkhäuser, 3 ed., 1967.

[12] R. Richter, Die Induktionsmaschinen, vol. 4 of Elektrische Maschinen. Basel, Stuttgart: Birkhäuser, 2 ed., 1954. 